\title{
Identification of the IMF sector structure in near-real time by ground magnetic data
}

\author{
A. S. Janzhura and O. A. Troshichev \\ Arctic and Antarctic Research Institute, St. Petersburg, Russia \\ Received: 7 February 2011 - Revised: 4 July 2011 - Accepted: 5 July 2011 - Published: 31 August 2011
}

\begin{abstract}
A method is proposed to determine in near-real time the interplanetary magnetic field (IMF) sector structure (SS) effect on geomagnetic data from polar cap stations. To separate the SS effect, whose polarity is invariant within an interval from some days to 2 weeks, from the disturbed solar wind effects with periodicity from minutes to hours, the daily median values of geomagnetic $\mathrm{H}$ (or D) component are estimated. Then the median values for 9 days preceding the current day are subjected to 3-days running averages and the interpolation procedure is applied to these smoothed averages. Comparisons of the sector structure reconstructed from the ground magnetic data with the actual variations of the GSM IMF $B_{\mathrm{y}}$ component measured onboard the ACE spacecraft in the summer months of 1990 and 2001 demonstrate their good agreement with coefficient of correlation $R=0.96-0.97$ for the H-component and $R=0.93-0.95$ for the D-component. The proposed simple method makes possible identification of the SS effect in the same near real-time regime as the derivation of the quiet daily curve and as level of reference for the polar cap magnetic disturbances in the calculation of the polar cap magnetic activity PC index.
\end{abstract}

Keywords. Interplanetary physics (Interplanetary magnetic fields) - Magnetospheric physics (Polar cap phenomena; Solar wind-magnetosphere interactions)

\section{Introduction}

A review of the methods applied for the derivation of the polar cap magnetic activity PC index was recently published by McCreadie and Menvielle (2010). The review describes calculation procedures used at the Arctic and Antarctic Research Institute (AARI) and Danish Meteorological Institute

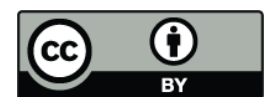

Correspondence to: O. A. Troshichev (olegtro@aari.nw.ru)
(DMI) to produce the unified PC index (Troshichev et al., 2006) and calls attention to the fact that the procedures applied in AARI and DMI are not identical and the PC indices routinely produced in DMI (PCN, northern) and AARI (PCS, southern) cannot be considered as unified indices. Indeed, the procedures are not fully identical, although they provide the geophysical community with very similar results. The main issue which is distinctly delineated in McCreadie and Menvielle (2010) is the question about the level of reference when estimating the magnetic disturbances value since other differences in the techniques are of secondary importance. The procedure applied in AARI takes into account the quiet daily curve (QDC) which is estimated for each day (see Janzhura and Troshichev, 2008). A method of calculating a running QDC makes it possible to determine a quiet daily variation even for magnetically disturbed days during solar maximum epochs and, correspondingly, to calculate the PC index in a quasi-real time regime. The procedure applied in DMI also uses a quiet daily variation as an hourly function of UT as a level of reference, but this quiet variation is derived as the mean of quiet variations for 5 previous years, or as a mean of quiet variations for months preceding the examined day.

One important detail in the procedure of the QDC derivation, which was not highlighted in Janzura and Troshichev (2008) and thus was not mentioned in McCreadie and Meanvielle (2010), is a problem of allowance for sector structure effects. The term "sector structure" (SS) denotes separation of the large-scale solar wind structure on an even number of sectors with opposite polarity of the interplanetary magnetic field (IMF) radial $B_{\mathrm{x}}$ component. The SS periodicity is determined by the solar corona magnetic field extending into the space and can remain unchanged during around solar rotations of $\sim 27$ days. However, the steady periodicity of the sector polarity alteration is observed only in epochs of the quiet Sun. In the growth and maximum solar activity epochs, the sector structure duration shortens dramatically

Published by Copernicus Publications on behalf of the European Geosciences Union. 
and becomes variable. The SS polarity in the GSM coordinate system is defined by the sign of the IMF radial $\left(B_{\mathrm{x}}\right)$ component. The SS is positive if $B_{\mathrm{X}}>0$ (usually, in combination with the negative tangential IMF component $B_{\mathrm{y}}<0$ ) and it is negative if $B_{\mathrm{x}}<0$ (and $B_{\mathrm{y}}>0$ ).

The ground magnetic disturbances related to the IMF sector structure were separated at first by Svalgaard (1968) and Mansurov (1969). Later it was demonstrated that the SSassociated magnetic disturbances are determined by variations of the IMF azimuthal component (Friis-Christensen et al., 1972; Sumaruk and Feldstein, 1973; Mishin et al., 1973). The multi-functional analysis of relationships between the polar cap magnetic disturbances and the IMF variations, representing the combination of radial $\left(B_{\mathrm{X}}\right)$, azimuthal $\left(B_{\mathrm{y}}\right)$ and vertical $\left(B_{\mathrm{z}}\right)$ components changes, has revealed that the current systems are related to the $B_{\mathrm{z}}$ and $B_{\mathrm{y}}$ components, whereas the radial $B_{\mathrm{x}}$ component is almost ineffective (Troshichev and Tsyganenko, 1979). The current system of magnetic disturbances DP2 related to the IMF southward $B_{\mathrm{ZS}}$ component consists of two vortices, roughly symmetrical relative to the Sun-Earth line (DP2 current system) (Nishida, 1968; Kuznetsov and Troshichev, 1977). The current system of disturbances related to the IMF $B_{\mathrm{y}}$ component includes currents flowing in the day-time cusp region along geomagnetic latitudes $\Phi \sim 78-80^{\circ}$, the current direction dependent on the IMF $B_{\mathrm{y}}$ sign (Friis-Christensen and Wilhjelm, 1975). It should be noted that disturbances related to the IMF $B_{\mathrm{y}}$ component are typical of the summer polar cap; in the winter season their intensity is usually negligible.

The DP2 current system and the corresponding electric fields in the polar ionosphere are generated by the magnetospheric field-aligned currents (FAC Region 1), flowing into the ionosphere on the poleward boundary of the auroral oval in the morning sector and flowing out of the ionosphere in the evening sector (Iijima and Potemra, 1976). The azimuthal IMF component controls the BY FAC system observed in the day-time cusp region during the summer season. The BY FAC system consists of two current sheets (Wilhjelm et al., 1978), one of which is located on the equatorward side of the cusp, i.e. in the Region 1, whereas another sheet is located on the poleward boundary of the cusp (Region 3), the direction of currents in the sheets being determined by the IMF $B_{\mathrm{y}}$ sign, as well as the direction of the ionospheric currents flowing between the current sheets (Friis-Christensen and Wilhjelm, 1975). Dependence of field-aligned currents on the IMF $B_{\mathrm{y}}$ component in the southern polar region is opposite to that in the Northern Hemisphere.

Thus, the IMF $B_{\mathrm{y}}$ component influence on the ground magnetic field in the polar cap is realized through generation of the BY FAC system in the day-time cusp region which leads to deformation of the regular ionospheric DP2 current systems. In the northern polar cap the morning current vortex is enhanced and expands into the dusk sector under conditions of IMF $B_{\mathrm{y}}>0$, and the evening vortex is enhanced and expands into the dawn sector under conditions $B_{\mathrm{y}}<0$
(Matsushita et al., 1973). In the southern polar region the asymmetry is reversed. Deformation of the current system displays as modification of the daily magnetic variation observed at the polar stations, which rotate together with the Earth under the current system fixed relative to the Sun.

The IMF $B_{\mathrm{y}}$ effect on the polar cap magnetic field consists of two parts concerning their time-scale: the first one is the sector structure effect, which is considered as invariable within the limits of the certain sector (within a time interval from a few days to 2 weeks), and the second one is the effect of the IMF short-time changes with periodicity from a few minutes to some hours. The "short-term" IMF fluctuations typical of the disturbed solar wind conditions are observed against the background of the "long-term" SS polarity changes. Since the short-term fluctuations are usually more powerful in magnitude than the sector structure effect, the SS influence is usually masked by the disturbed solar wind effect.

To clearly demonstrate the relationship between these two effects, Fig. 1 shows the actual variation of 1 -min values of the geomagnetic H-component observed at Thule station (THL) in the summer season of 2001 (thin lines) and the quiet daily curve (QDC) characterizing the daily variation of the quiet geomagnetic field (thick solid lines). One can see that the QDC level (daily averaged value of QDC) displays long-term changes, which are determined by the sector structure: the level rises under conditions of positive SS polarity and goes down under conditions of negative polarity. The SS effect at Thule station is comparable with the QDC amplitude. Both quantities are maximal $(\sim 100 \mathrm{nT})$ during the summer solstice owing to the high conductivity of the sunlit summer ionosphere. The periodicity of the sector structure in the summer season of 2001 was extremely variable (from 8 days to 27 days) and, therefore, it could not be designated a priori.

Thus, if we are going to analyze the polar cap magnetic activity produced by the IMF fluctuations related to disturbed solar wind, we have to exclude first the sector structure effect. This is not a serious problem if the analysis is carried out postfactum, based on long series of the ground magnetic observations and the IMF data obtained from spacecraft. However, the task turns out to be nontrivial while monitoring the actual magnetic activity, when the quiet daily curve (QDC) should be derived in quasi-real time (see Janzhura and Troshichev, 2008). In addition, as we see in Fig. 1, the actual periodicity can quickly alter (from 27 to 8 days), excluding the possibility of predetermining the SS effect.

In this paper we examine the possibility of identifying the SS effect in quasi-real time on the basis of only ground-based magnetic data. Although magnetic data from two polar cap stations Thule in the Northern Hemisphere and Vostok in the Southern Hemisphere are used for calculation of the PCN and PCS indices correspondingly, the analysis is carried out only for Thule, where the SS effect is much larger than that at Vostok. The reason of this peculiarity is, in the first place, 


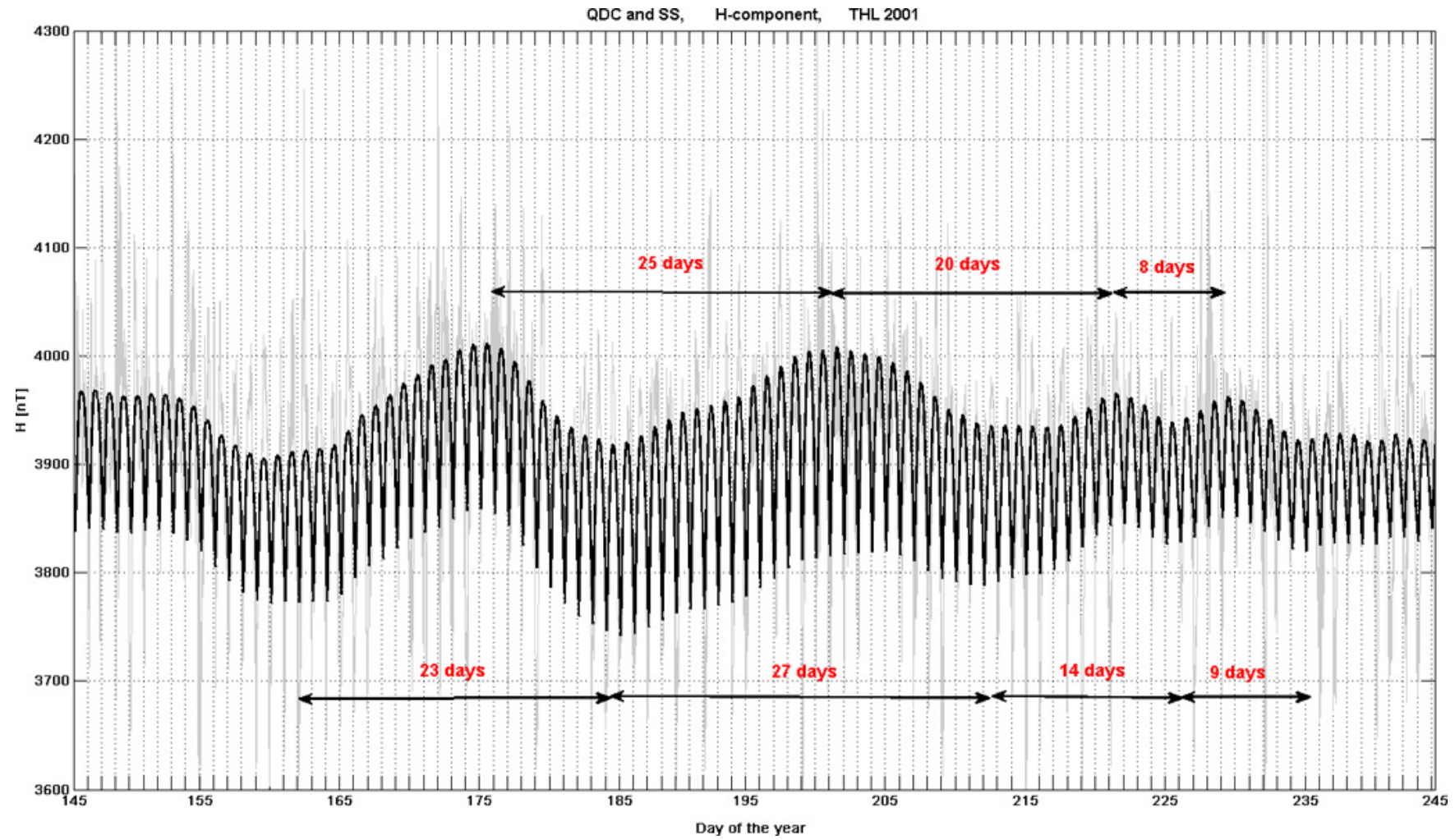

Fig. 1. Superposition of the actual variation of 1-min values of the geomagnetic H-component observed at Thule station in the summer season of 2001 (thin lines) and the quiet daily curve (QDC) characterizing the daily variation of the quiet geomagnetic field (thick solid lines).

nearly no ground-induced currents in the ice dome under the Vostok station; second, the disposition of Thule station, which is located at a higher geomagnetic latitude than Vostok. As a result, the SS effect at Vostok is negligible, as distinct from Thule where the SS effect is significant and its allowance is of crucial importance for the QDC derivation and correct evaluation of the $\mathrm{PCN}$ value. The data for years 1998 and 2001 near solar maximum have been taken for the analysis to test the method of allowing for the SS effect on the example of the most complex solar wind structures. IMF data were provided by measurements of solar wind parameters on board the ACE satellite (http://omniweb.gsfc.nasa.gov).

\section{Adequacy of the IMF sector structures derived from the ground-based and spacecraft observations}

How is the IMF sector structure derived from the groundbased magnetic observations? The interrupted long series of the experimental data were taken and the same method of filtration of data with a 7-days averaging window and nonlinear smoothing was used to separate the sector structure in the ground-based magnetic observations and spacecraft IMF measurements. The 7-day window was chosen to make allowance for the shortest period of the SS alteration. Reduc- ing the window width less than 7 days reflects in substantial deflections in the averaged curve owing to the irregular (short-term) disturbances in the solar wind. By increasing the window more than 7 days, the shorter periods in SS are essentially suppressed, although the regular 27-day periodicity keeps its true value. The method of filtration based on the regression analysis of the steadiest quantities within the window was applied. A robust version of a local regression using weighted linear least squares and the 1st-degree polynomial model was taken to bring down the weight of outliers in the regression: zero weight is given to data outside six mean absolute deviations. As an example, Fig. 2 shows the SS effect, displayed by this method (yellow line) in the data set of the IMF $B_{\mathrm{y}}$ measurements (blue line) on board ACE spacecraft for 1998 and 2001.

The IMF sector structure, determined by the IMF $B_{\mathrm{y}}$ component, leads to regular distortions of the DP2 current system where the morning or evening current circle is expanded and enhanced. As a result of the DP2 current system modification, the daily variation of the polar cap magnetic activity changes, so that the daily level of activity at a certain station either increases or decreases, being dependent on the sign of the sector structure. As soon as the SS sign changes (with period depending on the solar activity epoch), the mean value 

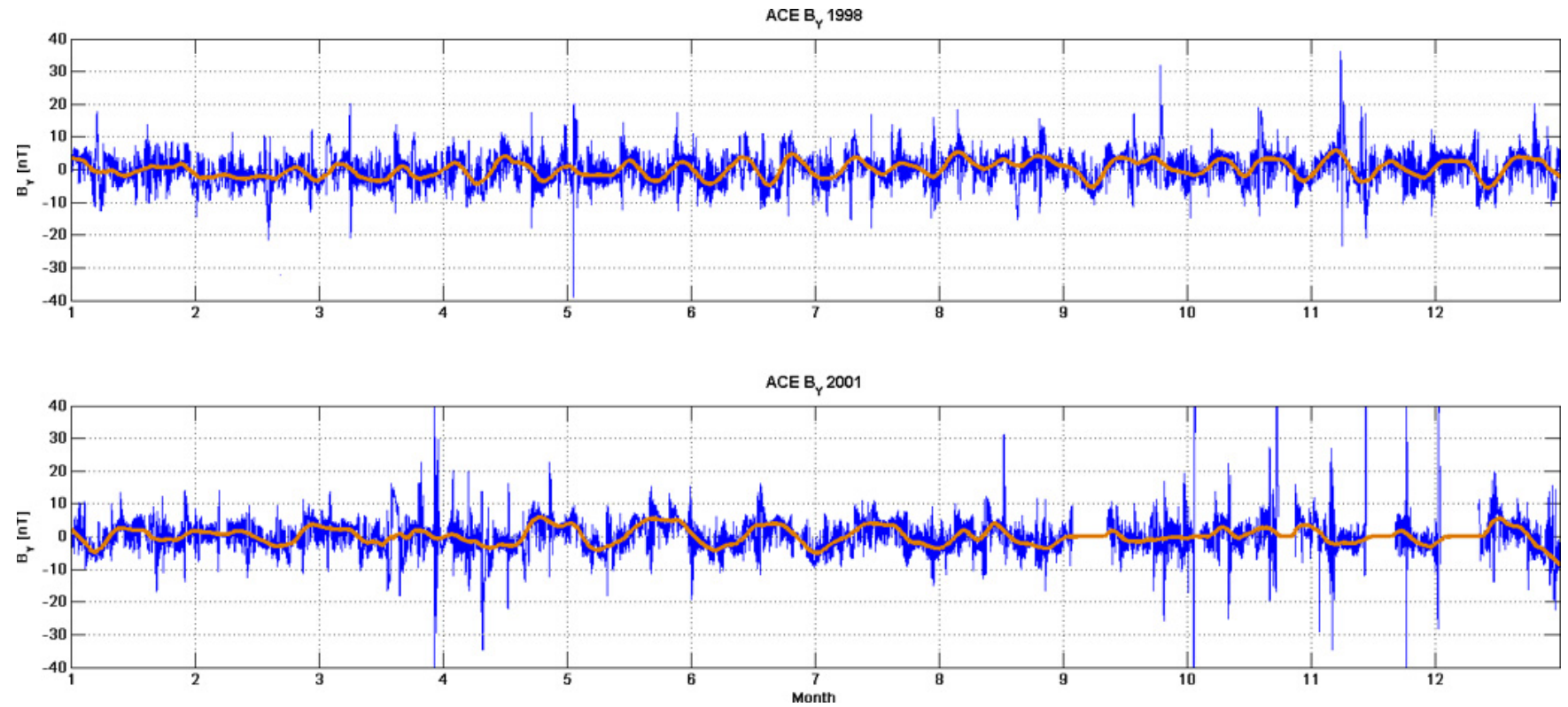

Fig. 2. The SS effect, displayed by the method of filtration with the 7-day averaging window and nonlinear smoothing (yellow line) and the original data series of the IMF $B_{\mathrm{y}}$ measurements on board ACE spacecraft (blue line) for 1998 and 2001.


Fig. 3. The SS effect, displayed by the method of filtration with the 7-day averaging window and nonlinear smoothing (yellow line) and the original data series of the ground magnetic $D$ and $H$ variations observed at station Thule (blue lines) for 1998 and 2001.

of the magnetic field periodically increases or decreases with the same period. Figure 3 shows the SS effect, displayed by the above described method (yellow line) in the data set of the ground magnetic $D$ and $H$ variations observed at Thule station (Greenland).

To clearly demonstrate consistency between SS structures displayed in the IMF $B_{\mathrm{y}}$ variations measured by spacecraft and ground magnetic variations, the coefficient of regression between these two quantities for the summer period was calculated and was close to 10 . Then mean values for both quantities in summer were determined and amplitudes of both quantities were normalized by these mean values, whereupon the mean quantities were reduced to zero. Figure 4 shows the results of the comparison for 1998 and 2001, the values 

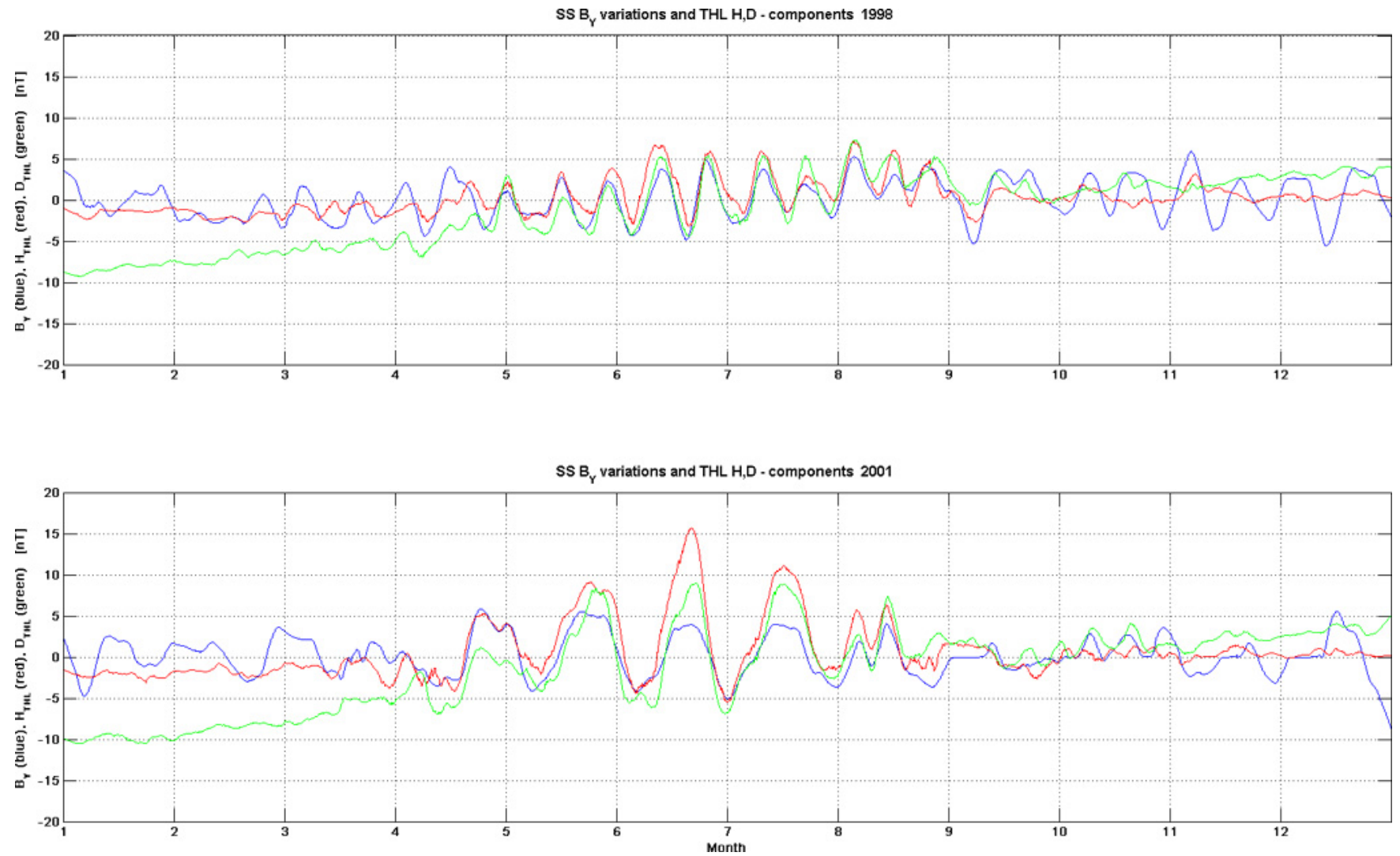

Fig. 4. Results of comparison between SS structures displayed in the IMF $B_{\mathrm{y}}$ variations (blue) measured by spacecraft and ground magnetic variations observed in $\mathrm{H}$ (red line) and D (green line) components at Thule for 1998 and 2001, where the mean values of all quantities were reduced to zero and the amplitudes were normalized.

of ground magnetic components $\mathrm{H}$ (red) and $\mathrm{D}$ (green) being divided by 10 for convenience of comparison. One can see that the SS effects derived by spacecraft measurements and ground-based magnetic observations are consistent during the summer season, when the magnetic disturbances generated by the IMF $B_{\mathrm{y}}$ component at Thule station are the most significant. The yearly drift of the magnetic D-component at Thule is due to the secular variation in the main geomagnetic field, which is very pronounced at this near-pole station.

\section{Identification of the SS effect by the ground magnetic data in quasi-real time}

Let us examine the averaged daily magnetic variations observed under conditions of positive and negative sector structure. Figure 5 presents, as an example, the mean daily variation in $\mathrm{H}$ magnetic component at station Thule derived for the summer months (May-August) of 1998 and 2001 under three gradations of the IMF azimuthal component $B_{\mathrm{y}}>3 \mathrm{nT}$ (red line), $B_{\mathrm{y}}<-3 \mathrm{nT}$ (green line) and $-2<B_{\mathrm{y}}<2 \mathrm{nT}$ (blue line). As Fig. 5 shows, the mean daily variation at Thule is displayed by the magnetic field increase around midnight (00:00-08:00 UT) and the magnetic field decrease around midday (12:00-20:00 UT), the magnitude of the daily changes being about $120 \mathrm{nT}$. Influence of the IMF $B_{\mathrm{y}}$ component is evident around local noon, when the H-component value strongly increases under conditions of positive SS structure $\left(B_{\mathrm{y}}>3 \mathrm{nT}\right)$ and strongly decreases under conditions of negative SS structure $\left(B_{\mathrm{y}}<-3 \mathrm{nT}\right)$, the magnitude of the SS effect being more than $150 \mathrm{nT}$. The SS effect in the night-time sector is negligible or irregular. These changes in the ground magnetic field are due to electric currents generated by the IMF $B_{\mathrm{y}}$ component in the day-time cusp region, the current direction being defined by sign of $B_{\mathrm{y}}$. Thus, it will suffice to estimate the daily magnetic field deviation relative to the mean daily variation for conditions of $B_{\mathrm{y}} \sim 0$ (blue line) to identify the SS effect providing that the solar wind is undisturbed. The appropriate difference between the daily averaged and daily median values will determine at once the sign and intensity of the SS effect.

If the solar wind is disturbed, the SS effect is superposed with the effect of the solar wind fluctuations, where the latter might be larger than the SS effects. As a result, the daily averaged value of the magnetic field can display the disturbance level rather than the SS effect. Taking into account the 

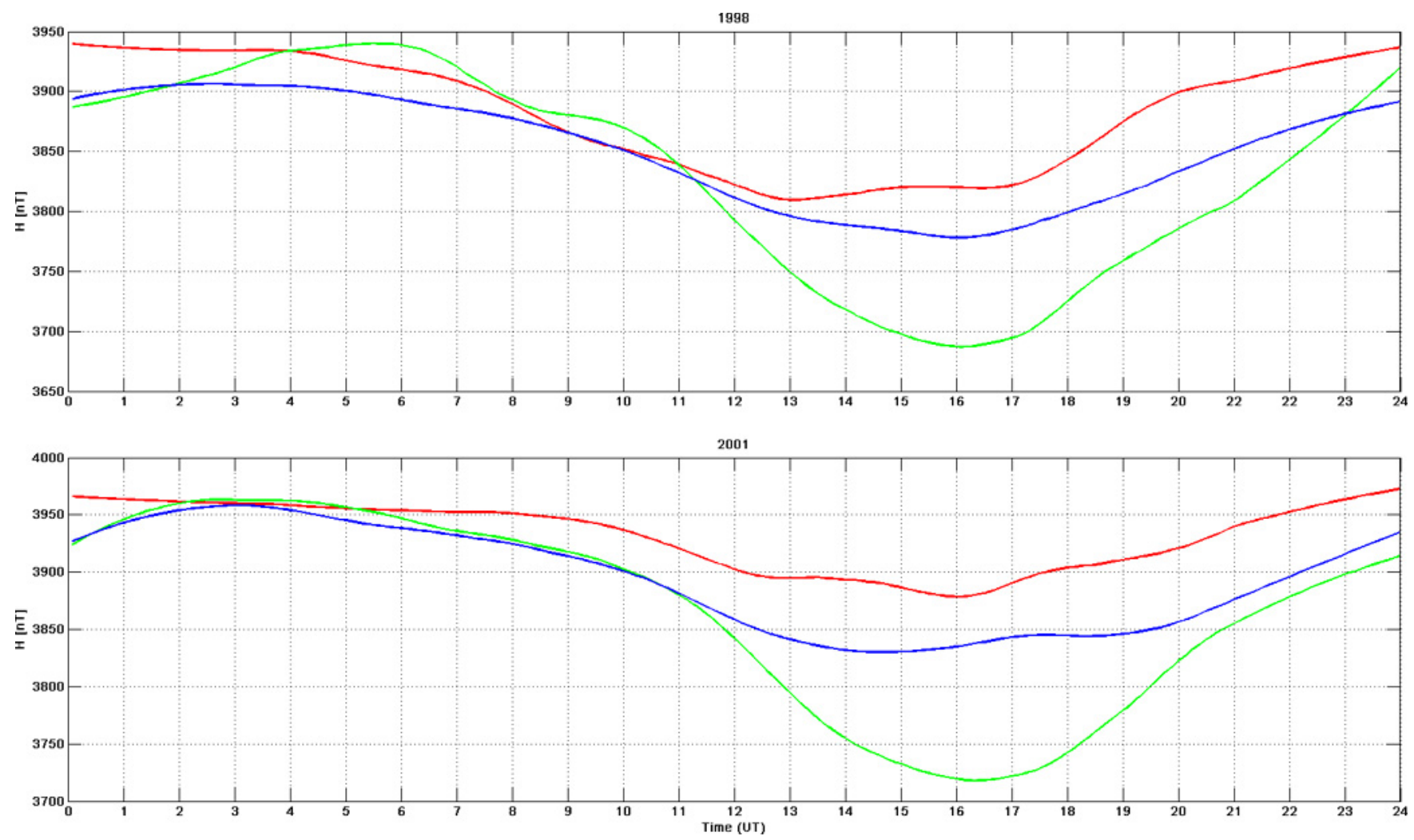

Fig. 5. The mean daily variation in $\mathrm{H}$ magnetic component at station Thule derived for three gradations of the IMF azimuthal component $B_{\mathrm{y}}>3 \mathrm{nT}$ (red line), $B_{\mathrm{y}}<-3 \mathrm{nT}$ (green line) and $-2<B_{\mathrm{y}}<2 \mathrm{nT}$ (blue line) for the summer months (May-August) of 1998 and 2001.

quite different length of the sector structure (days) and the disturbed wind fluctuations (hours) we have to give attention in these cases to the daily median values for the previous days. It is evident that the interval of examination should not be longer than the minimal duration of sector structure (i.e. few days), otherwise the SS effect is smoothed. The optimal interval was revealed experimentally.

Figure 6 shows the behavior of the median values of the magnetic $\mathrm{H}$-component at Thule station during June months of 1998 and 2001 for intervals with duration of 1 day (blue line), 3 days (green line), and 5 days (black line). The run of the IMF $B_{\mathrm{y}}$ component, derived from spacecraft measurements by the method described in Sect. 2, is shown by a dotted red line, the actual $B_{\mathrm{y}}$ values being raised to the ground magnetic scale by a magnification of 5 times and shifted by $50 \mathrm{nT}$ to a higher position for a clear comparison. One can see that the disturbed solar wind effects are strongly dominant in the case of the 1-day running average. On the other end the 5-day running average (black line) strongly smoothes not only the effect of irregular disturbances but the SS effect as well, especially for periods of short sector structures (June 1998). The 3-day running average of median values (green curve) seems to be the optimal to provide information on sign and level of the SS effect, but this information could be also distorted, if the strongly disturbed days are involved in the analysis.

Keeping in mind this specification, the 3-day smoothing averages of the median values were subjected to the interpolation procedure including the following steps:

1. median values for magnetic components $H$ and $D$ are derived for 4 intervals of preceding days with the exception of the current day $(n=0)$ :

- r1 = F [for interval from $n-3$ day to $n-1$ day]

$-\mathrm{r} 2=\mathrm{F}$ [for interval from $n-5$ day to $n-3$ day]

$-\mathrm{r} 3=\mathrm{F}$ [for interval from $n-7$ day to $n-5$ day]

- r4 = F [for interval from $n-9$ day to $n-7$ day];

2. piecewise polynomial form of the cubic spline interpolant for $\mathrm{r} 1, \mathrm{r} 2, \mathrm{r} 3$, and $\mathrm{r} 4$ segments is determined;

3. termination of this form related to day $n=0$ is examined as representative of the SS effect for the current day $n=$ 0 , even if this day is disturbed.

The procedure is repeated each subsequent day. Results of the procedure, the variation of the reconstructed magnetic $\mathrm{H}$ component, are presented by the magenta line in the same 

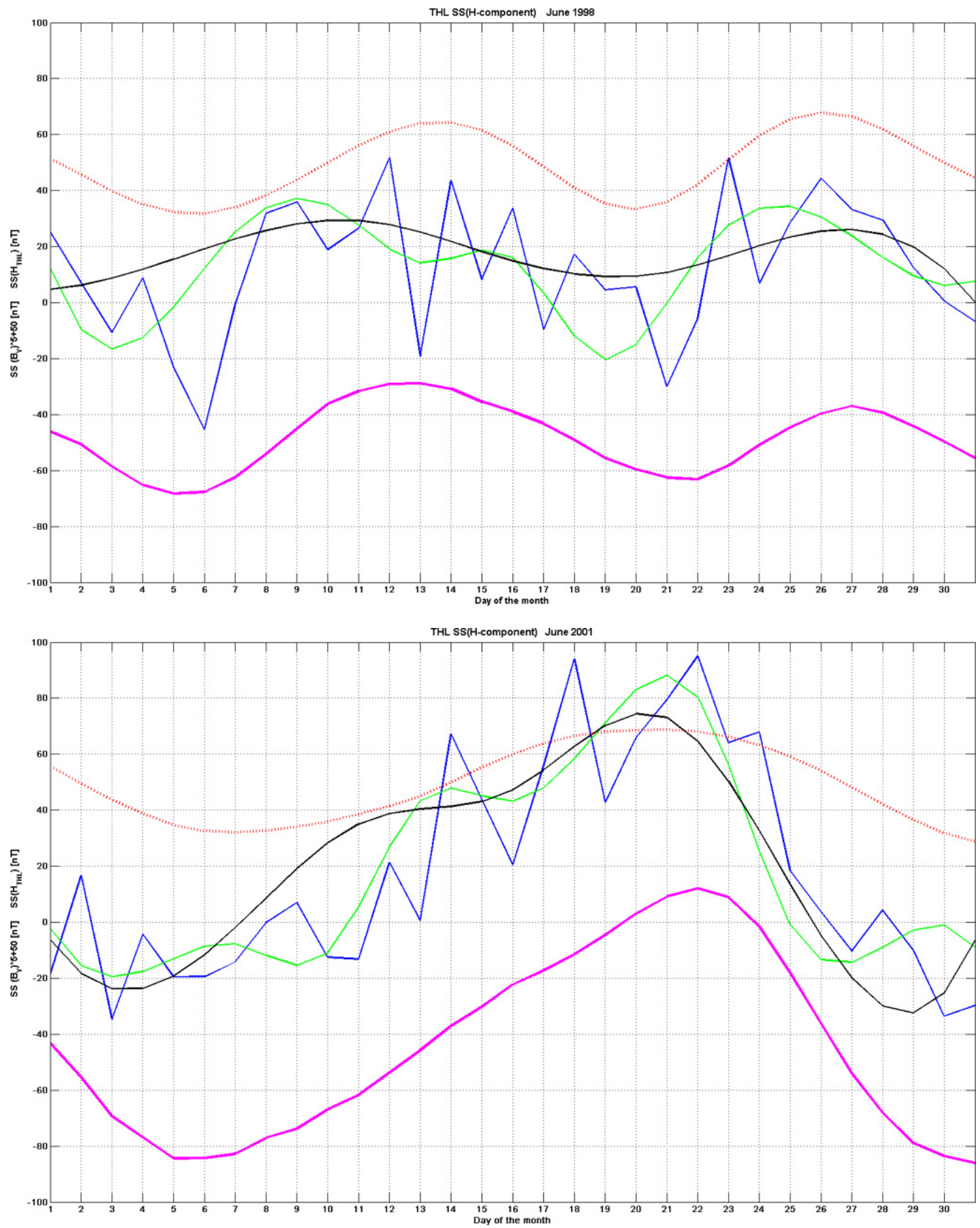

Fig. 6. Behavior of the median values of the magnetic H-component at Thule station during June months of 1998 (a) and 2001 (b) for intervals with duration of 1 day (blue line), 3 days (green line), and 5 days (black line). The red dotted line shows the variation of the IMF $B_{\mathrm{y}}$ component, derived from spacecraft measurements. The magenta line shows the variation of the reconstructed magnetic H-component. To be clearly demonstrated, the actual $B_{\mathrm{y}}$ values were multiplied by five and were shifted by $50 \mathrm{nT}$ to a higher position, whereas the curve of reconstructed $\mathrm{H}$-component was shifted by $50 \mathrm{nT}$ to a lower position.

Fig. 6, the reconstructed H-component curve being shifted by $50 \mathrm{nT}$ to a lower position. Comparison of the red and magenta lines shows their good consistency in phase with the SS effect and quite satisfactory consistency in the amplitude of the SS effect.
Results of the SS structure identification by the data of the $\mathrm{H}$-component observed at station Thule for the entire years of 1998 and 2001 are shown in Fig. 7. The green line is the actual variation of the H-component; the black asterisks present the interpolated SS structure derived by the above 

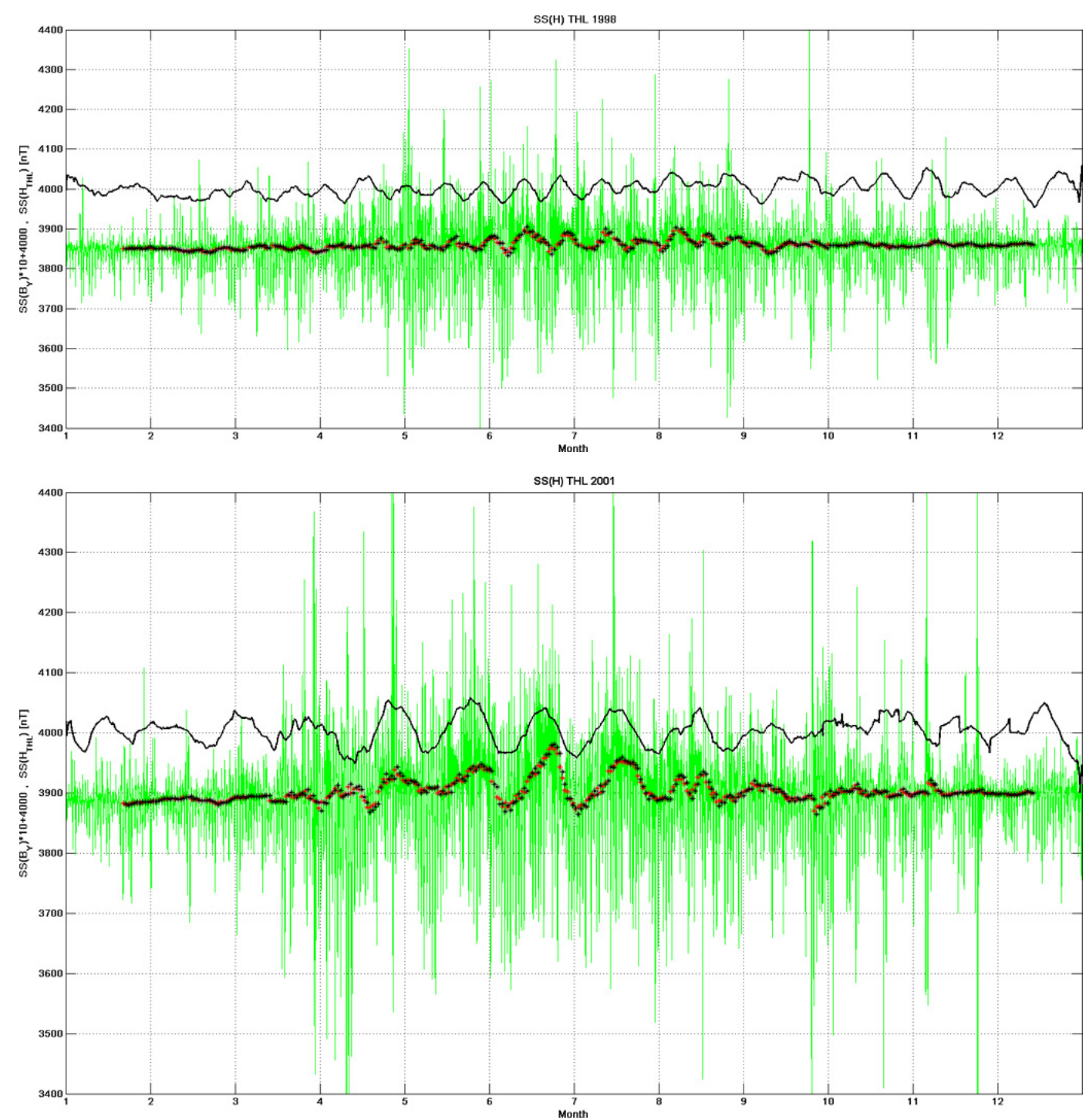

Fig. 7. The SS effect derived in the H-component observed at station Thule in 1998 (a) and 2001 (b). The actual variation of the ground Hcomponent is shown by the green line, whereas black asterisks present the extrapolated SS structure obtained by the extrapolation procedure when all data are available till the examined day $(n=0)$, and red asterisks present the interpolated SS structure derived under the condition that the examined day is in the middle of a gap in the time interval. The actual variation of the IMF $B_{\mathrm{y}}$ component, measured by ACE spacecraft, is shown by the thin black line.

described interpolation procedure under the condition that data are available up to the examined day $(n=0)$; the red asterisks present the interpolated SS structure derived under conditions that the examined day is a middle point in an extrapolated interval. The actual variation of the IMF $B_{\mathrm{y}}$ component, measured by ACE spacecraft is shown by a thin black line.

To verify the proposed method, we examined the relationship between the daily values of actual IMF $B_{\mathrm{y}}$ quantities measured by the ACE spacecraft and interpolated SS quantities derived from geomagnetic variations in $\mathrm{H}-$ and D-components at Thule station. The analysis was carried out for two summer months (June-July of 1998 and 2001), when the SS effect in the polar cap magnetic variations is most pronounced. The value of the effect decreases away from summer solstice and falls to zero in the winter season because of the low conductivity of the winter ionosphere. The results presented in Fig. 8 show a good correlation between the ground and satellite sets of data on sector structure; the geomagnetic H-component ensures a little better correlation ( $R=0.96-0.97)$ than the geomagnetic D-component $(R=0.93-0.95)$. Thus, the magnetic data at Thule display the same sector structure as measurements on board the ACE satellite. 

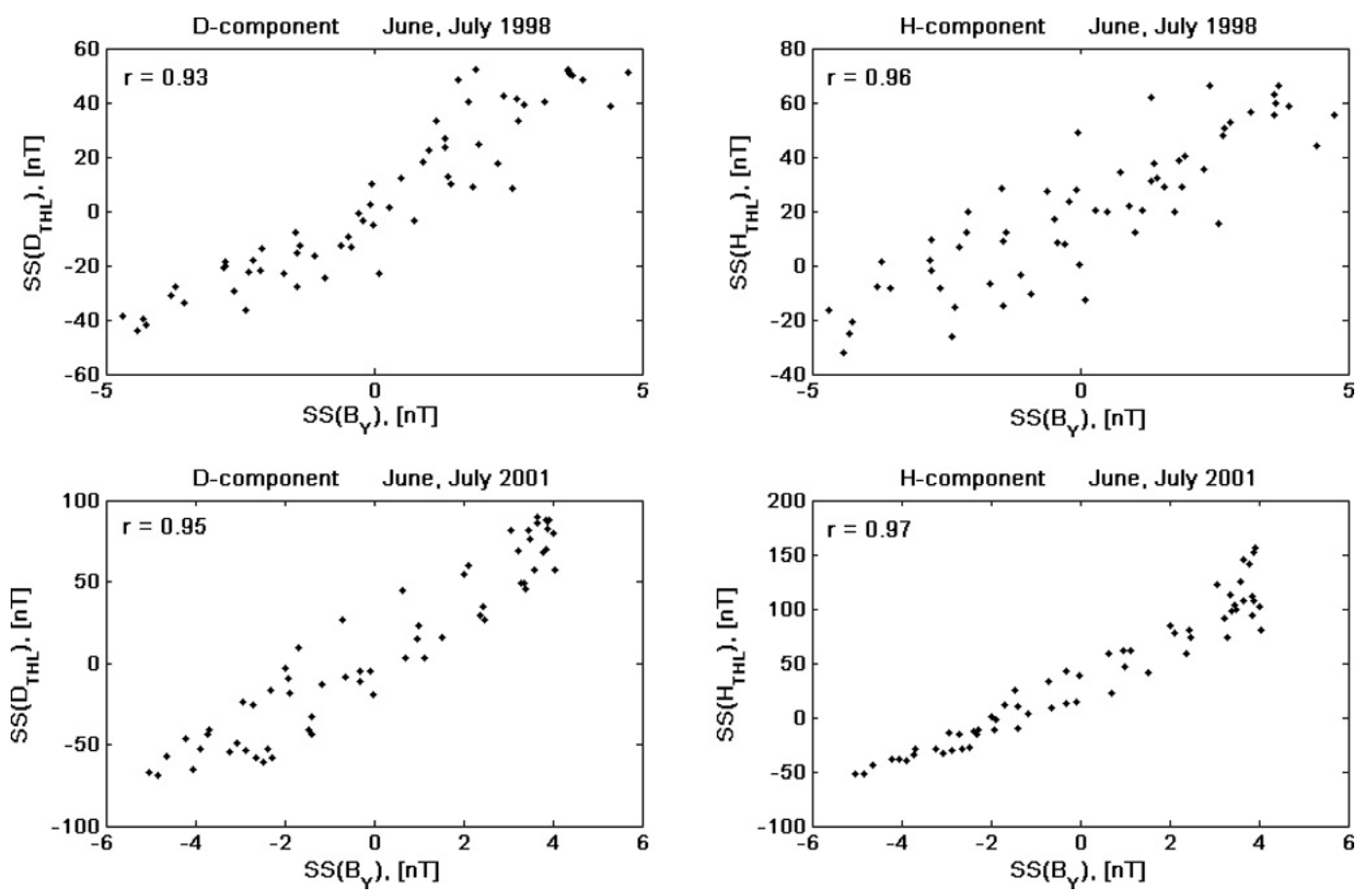

Fig. 8. The relationship between the satellite and ground-based sets of magnetic data on variations caused by the IMF sector structure in the summer months of 1998 (upper row) and 1991 (lower row) for geomagnetic D (left column) and H (right column) components at Thule.

\section{Discussion and conclusions}

Troshichev et al. (2006) suggested calculating the PC index in quasi-real time. With this aim, a special procedure was elaborated to determine for each day the quiet daily curve (QDC) used as a line of reference for values of the ground magnetic disturbance (see description in Janzhura and Troshichev, 2008). The procedure of QDC derivation implies consideration of the quiet magnetic field for quiet days or quiet periods for different days provided that the quiet times are related to a single level of reference. This level of reference can be derived if the SS effect is taken into account prior to the QDC derivation. It is especially important for Thule station where the SS effect can be much higher than the QDC amplitude.

It is well known that the SS effect is displayed in the best way in variations (increase or decrease) of the vertical (Z) component of geomagnetic field in the near-pole region. However, using the geomagnetic Z-component we have to deal with strong effect of a global magnetic storms, which are generated by the ring currents developed in the inner magnetosphere. The ring current (DR) effect is displayed as a strong depression of geomagnetic H-component in the low-latitude zone. In the high-latitude area the DR effect is displayed as a strong increase of the positive (downward) Zcomponent in the northern polar cap, and increase of the negative (upward) Z-component in the southern polar cap, the influence on geomagnetic $\mathrm{H}$ - and D-component being negligible. Taking into account that magnetic storms can last some days with unpredictable changes in their activity, the SS effect identification using the geomagnetic Z-component can be a very complicated task under storm conditions. Naturally, the satellite measurements is the most direct way to the SS effect identification, however, in such a case an additional problem arises how to correctly convert the satellite information about the IMF $B_{\mathrm{y}}$ component into the SS effect in the geomagnetic $H$ and $D$ variations.

Since disturbances in geomagnetic $\mathrm{H}$ - and D-components form the basis for derivation of the PC index, allowance for $\mathrm{SS}$ effect in $\mathrm{H}$ - and $\mathrm{D}$-components is the most reliable way for derivation of the QDC as a level of reference to estimate the value of disturbances. The method proposed in this paper makes possible identification of the SS effect in the same time regime as that for the QDC derivation, magnetic disturbance value evaluation and PC index calculation, i.e. in quasi-real time. At present it takes only a few minutes for transmitting and processing the data from the point of observation (Vostok, for example) to the Center (AARI) and publishing the PC index at the AARI website.

Although this paper is mostly concerned with how IMF $B_{\mathrm{y}}$ affects the $\mathrm{H}$ and $\mathrm{D}$ magnetic ground components of Thule in summer, our methods could also be used to estimate IMF $B_{\mathrm{y}}$ in summer from the ground magnetic data during periods when satellite IMF observations are missing. Since IMF $B_{\mathrm{y}}$ and $B_{\mathrm{x}}$ components are usually coupled within the Parker spiral, the method can be applied to reconstruct the IMF sector structures in pre-satellites epochs. 
Acknowledgements. Topical Editor R. Nakamura thanks B. Emery and C. Meng for their help in evaluating this paper.

\section{References}

Friis-Christensen, E. and Wilhjelm, J.: Polar cap currents for different directions of the interplanetary magnetic field in the Y-Z plane, J. Geophys. Res., 80, 1248-1260, 1975.

Friis-Christnsen, E., Lassen, K., Wilhjelm, J., Wilcox, J. M., Gonzalez, W., and Colburn, D. S.: Critical component of the interplanetary magnetic field responsible for large geomagnetic effects in the polar cap, J. Geophys. Res., 77, 3371-3376, 1972.

Iijima, T. and Potemra, T. A.: The amplitude distribution of fieldaligned currents of northern high latitudes observed by Triad, J. Geophys. Res., 81, 2165-2174, 1976.

Janzhura, A. S. and Troshichev, O. A., Determination of the running quiet daily geomagnetic variation, J. Atmos. Solar-Terr. Phys., 70, 962-972, 2008.

Kuznetsov, B. M. and Troshichev, O. A.: On the nature of polar cap magnetic activity during undisturbed periods, Planet. Space Sci., 25, 15-21, 1977.

Mansurov, S. M.: A new evidence for relationship between the space and earth magnetic fields, Geomagn. Aeronomy, 9, 768770, 1969 (in Russian).

Matsushita, S., Tarpley, J. D., and Campbell, W. H.: IMF sector structure effects on the quiet geomagnetic field, Radio Sci., 8, 963-972, 1973.
McCreadie, H. and Menvielle, M.: The PC index: review of methods, Ann. Geophys., 28, 1887-1903, doi:10.5194/angeo28-1887-2010, 2010.

Mishin, V. M., Bazarzhapov, A. D., Nemtsova, E. I., Popov, G. V., and Shelomentsev, V. V.: Influence of interplanetary magnetic field on magnetospheric convection and electric field in ionosphere, Preprint SibIZMIRAN, N 5-73, 37 p., 1973 (in Russian).

Nishida, A.: Coherence of geomagnetic DP2 fluctuations with interplanetary magnetic variations, J. Geophys. Res., 73, 5549-5559, 1968.

Sumaruk, P. V. and Feldstein, Ya. I.: Seasonal variations of the geomagnetic $\mathrm{Z}$ component changes in the near-pole region and their relation to sign of the $Y_{\mathrm{SE}} \mathrm{IMF}$ component, Geomagn. Aeronomy, 13, 545-546, 1973 (in Russian).

Svalgaard, L.: Sector structure of the interplanetary magnetic field and daily variation of the geomagnetic field at high latitudes, Det Danske meteorologiske institute Charlottenlund, preprint R6, 1968.

Troshichev, O. A. and Tsyganenko, N. A.: Correlation relationships between variations of IMF and magnetic disturbances in the polar cap, Geomagn. Research, N25, 47-59, 1979 (in Russian).

Troshichev, O., Janzhura, A., and Stauning, P.: Unified PCN and PCS indices: Method of calculation, physical sense and dependence on the IMF azimuthal and northward components, J. Geophys. Res., 111, A05208, doi:10.1029/2005JA011402, 2006.

Wilhjelm, J., Friis-Christensen, E., and Potemra, T. A.: The relationship between ionospheric and field-aligned currents in the day-side cusp, J. Geophys. Res., 83, 5586-5594, 1978. 